\title{
Overcoming barriers to evidence-based patient blood management: a restricted review
}

\author{
Alana Delaforce ${ }^{1,4^{*}} \mathbb{D}$, Jed Duff', Judy Munday ${ }^{2,3}$ and Janet Hardy ${ }^{4}$
}

\begin{abstract}
Background: Blood transfusions are associated with a range of adverse patient outcomes, including coagulopathy, immunomodulation and haemolysis, which increase the risk of morbidity and mortality. Consideration of these risks and potential benefits are necessary when deciding to transfuse. Patient blood management (PBM) guidelines exist to assist in clinical decision-making, but they are underutilised. Exploration of barriers to the implementation and utilisation of the PBM guidelines is required. This study aimed to identify common barriers and implementation strategies used to implement PBM guidelines, with a comparison against current expert opinion.

Methods: A restricted review approach was used to identify the barriers to PBM guideline implementation as reported by health professionals and to review which implementation strategies have been used. Searches were undertaken in MEDLINE/PubMed, CINAHL, Embase, Scopus and the Cochrane library. The Consolidated Framework for Implementation Research (CFIR) was used to code barriers. The Expert Recommendations for Implementing Change (ERIC) tool was used to code implementation strategies, and subsequently, develop recommendations based on expert opinion.
\end{abstract}

Results: We identified 14 studies suitable for inclusion. There was a cluster of barriers commonly reported: access to knowledge and information $(n=7)$, knowledge and beliefs about the intervention $(=7)$ and tension for change $(n=6)$. Implementation strategies used varied widely $(n=25)$. Only one study reported the use of an implementation theory, model or framework. Most studies $(n=11)$ had at least 50\% agreement with the ERIC recommendations.

Conclusions: There are common barriers experienced by health professionals when trying to implement PBM guidelines. There is currently no conclusive evidence to suggest which implementation strategies are most effective. Further research using validated implementation approaches and improved reporting is required.

Keywords: Patient blood management, Consolidated Framework for Implementation Research, Expert Recommendations for Implementing Change, Barrier Mapping, Implementation Strategies

\footnotetext{
* Correspondence: Alana.delaforce@mater.org.au

${ }^{1}$ The University of Newcastle, School of Nursing and Midwifery, University

Drive, Callaghan, NSW 2302, Australia

${ }^{4}$ Mater Health Services, Level 6, Duncombe Building, Raymond Terrace, QLD

4101, Australia

Full list of author information is available at the end of the article
}

(c) The Author(s). 2020 Open Access This article is distributed under the terms of the Creative Commons Attribution 4.0 International License (http://creativecommons.org/licenses/by/4.0/), which permits unrestricted use, distribution, and reproduction in any medium, provided you give appropriate credit to the original author(s) and the source, provide a link to the Creative Commons license, and indicate if changes were made. The Creative Commons Public Domain Dedication waiver (http://creativecommons.org/publicdomain/zero/1.0/) applies to the data made available in this article, unless otherwise stated. 


\section{Contributions to the literature}

- Our review is the first to provide synthesised evidence regarding the barriers to patient blood management (PBM) guidelines.

- Our review reports implementation strategies used, then classifies and compares them against the Expert Recommendations for Implementing Change (ERIC) tool.

- Our review confirms that the reporting of implementation methods and implementation strategies used to enhance the uptake of PBM guidelines is currently limited and makes recommendations on how to improve the reporting of future studies.

\section{Background}

Blood transfusions carry significant risks to patient safety and should be used sparingly [1, 2]. Such risks include immunomodulation (where the patient acquires new antibodies, making it harder to locate compatible blood products), coagulopathies (increased risk of venous thromboembolism and pulmonary embolism), haemolysis (red cell destruction) and adverse reactions (including transfusion-associated circulatory overload and lung injury) [1-3]. Given the risk of morbidity and mortality associated with blood transfusions, it is crucial that patients only receive blood transfusions where the potential benefit outweighs these risks.

Globally, patient blood management (PBM) guidelines have been developed to provide clarity and support to clinicians when considering transfusion [4-7]. The guidelines consider three key principles, or "pillars" when making recommendations: the maximisation of a patient's red cell mass before invasive procedures, the minimisation of intraoperative blood loss and that patients are supported to tolerate anaemia rather than receive a blood transfusion [8, 9]. When implemented effectively, the guidelines can have a significant impact on improved patient care $[8,10,11]$. A systematic review published in 2018 found that implementation of a multimodal PBM program (using the three pillars) resulted in a $39 \%$ reduction in transfusion rates, in addition to statistically significant reductions in hospital length of stay and an overall reduction of $11 \%$ in mortality rates [8].

Many implementation strategies that support the implementation of PBM guidelines have been developed and utilised, but it is not clear which are the most effective [10-24]. Some examples of implementation strategies used to improve the uptake of PBM guidelines include using local consensus processes, audit and feedback, providing education and identifying and preparing champions [10-23]. A systematic review by
Tinmouth and colleagues found the use of behavioural implementation strategies to be effective at reducing blood product utilisation, but due to heterogeneity across studies, they could not make specific recommendations [25]. These difficulties are not unique to PBM guideline implementation, and much research has been undertaken to help advance the language, processes used and reporting of experiences to help provide clarity and direction to improve the translation of evidence to practice [26, 27].

There are several frameworks available in the literature that health professionals can use to identify barriers, guide intervention selection and support the implementation process [26, 28-30], such as the Consolidated Framework for Implementation Research(CFIR) that is utilised in this review [29]. The CFIR was developed to provide a unified taxonomy of existing frameworks and was the result of a systematic review of 19 existing frameworks [29]. The CFIR comprises five domains, and 39 theoretical constructs thought to influence implementation [31, 32]. The five domains include the intervention, the inner setting, outer setting, individuals involved and the process by which implementation is accomplished [29]. The CFIR also provides a comprehensive data dictionary that specifies what each construct means to assist with correct coding [29]. On its own, the CFIR is useful, but historically, it was not easily mapped to other tools to assist with implementation strategy selection, following barrier identification. A recently developed tool: Expert Recommendations for Implementing Change (ERIC) helps to address this limitation [33]. The ERIC implementation strategy selection tool comprises 73 strategies to enhance implementation [34]. The implementation strategies were compiled by 71 experts over three Delphi rounds in an attempt to gain consensus on what implementation strategies positively influence implementation [34]. The definitions of the implementation strategies are also outlined in a data dictionary to help guide correct classification [34]. The ERIC tool allows the user to select the relevant local barriers (as classified by the CFIR) and generate a list of implementation strategies that, according to expert opinion, should be effective in addressing them [33]. The ERIC tool is one of the many options that can be used to understand implementation problems. To date, the reported use of such frameworks and tools to guide implementation of patient blood management guidelines has been limited [33].

This review will examine implementation strategies used to address barriers to implementing patient blood management guidelines. Specifically, it aims to highlight the barriers identified by health professionals and any implementation strategies used. These are then compared against current expert opinion, based on the 
assumption that better selection of implementation strategies leads to improved translation of evidence into practice.

\section{Methods}

\section{Approach}

We utilised a newly described restricted systematic review approach, as proposed by Plüddeman and colleagues [35]. In the context of limited resources, the restricted review approach uses a flexible framework to select the level of rigour at each phase of the review [35]. The level of rigour is determined by the level of input from the team. For example, in a traditional review, two members are responsible for the title and abstract screening, whereas, in a restricted review, these may be undertaken by one author only [35]. In keeping with this method, we used pilot sampling during screening, study selection and quality assessment phases. An overview of the process is shown in Fig. 1.

\section{Searches}

We searched for publications that had the word "blood"

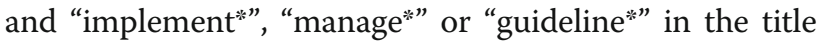
and excluded irrelevant terms (such as sugar, glucose, pressure and cholesterol). We included published literature only, in the Cumulative Index for Nursing and
Allied Health Literature (CINAHL), Embase (Ovid interface, 1948 onwards), MEDLINE (Ovid interface, 1948 onwards), Scopus and Cochrane library database. The initial search was undertaken in March 2018 and repeated in June 2019 to confirm there were no new relevant articles. We also hand-searched further articles by scanning references lists of full-text articles. After the removal of duplicates, one author completed the title and abstract screening in Covidence ${ }^{\mathrm{Tw}}$ (See Fig. 1. ).

\section{Study selection and data extraction}

Articles were eligible for inclusion if they were a primary research study of any design comparing PBM implementation strategies with usual or standard care, had identified barriers to implementing PBM guidelines and had been written in English and published between 1999 and 2019. This date range was chosen as the landmark study highlighting the risks associated with blood transfusions was published in 1999 [36]. We defined barriers as existing impediments to the uptake of the PBM guidelines. During the initial full-text screening, the second and third authors were blinded to the first author's decision. Resolution by consensus occurred where there was disagreement at this point, and the pilot screening process revealed the need for tighter inclusion criteria (finalised as per above). The amended criteria were then applied

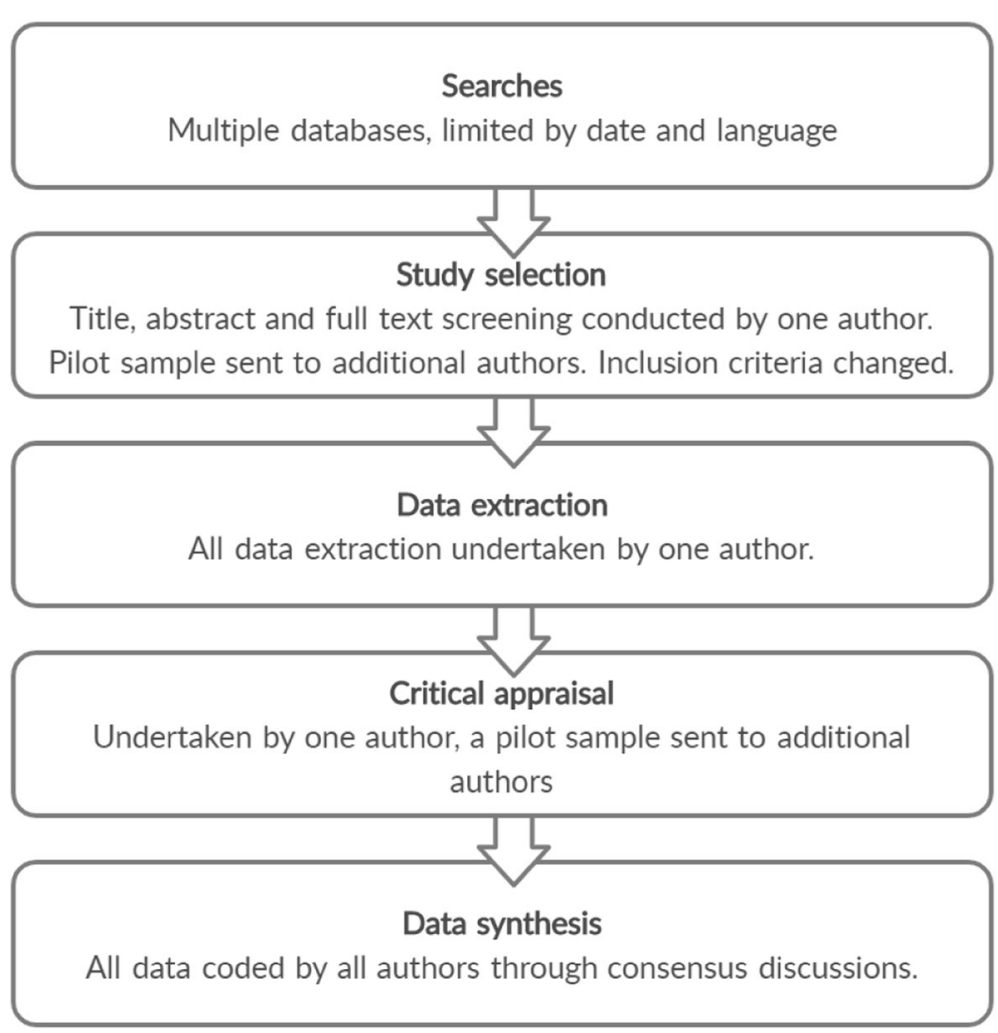

Fig. 1 Process of restricted review 
to all remaining articles. Post hoc adjustments to inclusion criteria are acceptable in restricted reviews such as this during pilot screening, when additional authors are reviewing full texts, and consensus discussions are taking place [35, 37, 38]. The PRISMA flow diagram [39] included details of the characteristics of excluded studies (Fig. 2). Data were extracted by one author (AD) using an online data extraction form. We collected demographic data, including geographic location, patient population, study design, research methods, barriers and implementation strategies used (Table 1). We also collected the reduction in red cell utilisation but did not undertake a full analysis as this has been addressed in a previous systematic review by Tinmouth and colleagues [25].

\section{Quality assessment}

The Mixed Methods Appraisal Tool (MMAT) was used for quality assessment as this facilitates rapid concurrent quality assessment across qualitative, quantitative and mixed methods studies [40]. The MMAT tool has two screening questions and four criteria (three for mixed methods studies) that the user nominates as being present or absent in each article [40]. For each criterion present, a score of $25 \%$ is awarded to the study. If all four criteria are met, then a score of $100 \%$ is assigned. Criteria are designed to gauge the reliability of the information and assess sample sizes, measurements used and whether there was a complete dataset [40].

\section{Data synthesis and presentation}

Data extracted were exported into an Excel ${ }^{\mathrm{Tw}}$ spreadsheet and collated into tables to facilitate the coding of barriers, implementation strategies and agreement with the ERIC tool recommendations [33]. The CFIR framework supported the classification and coding of barriers [29], and the ERIC classification tool supported implementation strategy coding [33]. Both associated data dictionaries provided coding guidance [29, 33, 34]. Multiple coding and classification of individual statements occurred where necessary. Consensus discussions between all three reviewers facilitated the full coding agreement.

Details of the implementation strategies used in each study to address identified barriers and the agreement with the ERIC tool for each paper are provided in Table 2. The barriers from each study were entered into the ERIC tool,



Fig. 2 Prisma flow diagram 


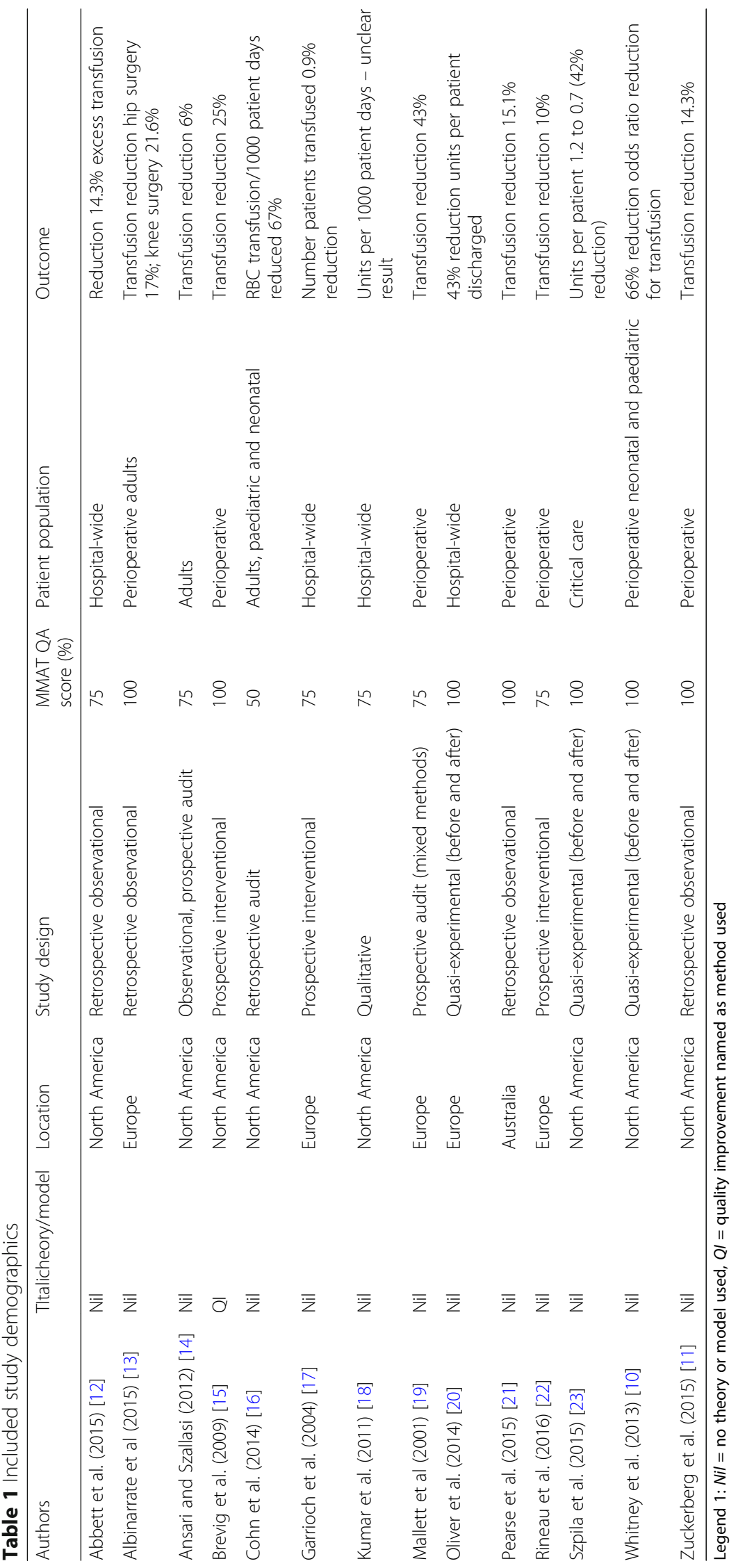




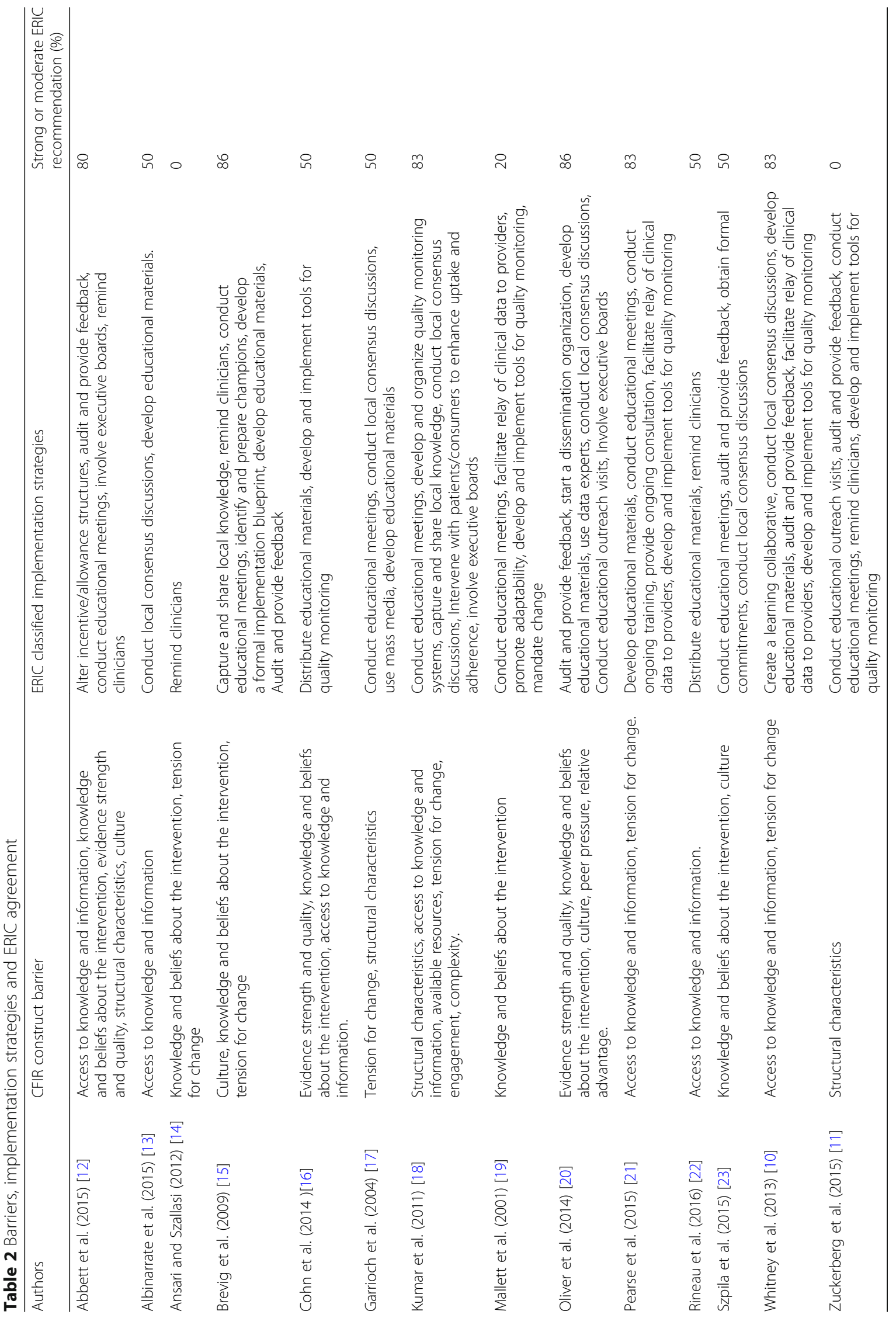


which provides a list of recommended implementation strategies based on the barrier selection made [33]. The ERIC tool provides categories for recommendations from weak, moderate, and strong. Strong recommendations are those with over $50 \%$ expert consensus that the implementation strategy is appropriate for a given barrier, and moderate are those with a 20 to $49 \%$ consensus [33]. Agreement with the ERIC recommendations was calculated based on overall barriers present and whether or not a moderate or strong recommendation for each implementation strategy used was evident. Table 3 provides details of all the barriers, the ERIC recommended implementation strategies and highlights in italic text which recommended strategies were used to address specific barriers.

\section{Results}

\section{Characteristics of eligible studies}

Fourteen papers were selected for final inclusion (see Table 1) [10-23]. Study designs included before and after implementation studies $(n=3)[10,20,23]$, retrospective observational $(n=6)[11-13,16,18,21]$ and prospective interventional studies $(n=5)[14,15,17,19,22]$. The majority of studies were conducted in Europe $(n=5)$ $[13,17,19,20,22]$ or North America $(n=8)[10-12,14-$ $16,18,23]$ with one paper from Australia [21]. Half of the included papers studied perioperative patient populations $(50 \%, n=7)[10,11,13,15,19,21,41]$, while $7 \%(n=1)$ were focused on critical care [23], and $43 \%(n=6)$ were unspecified $[12,14,16-18,20]$. Outcome measures/results were reported in multiple formats. The majority $(64 \%, n=$ 9) $[10,12-15,19,21-23]$ reported crude reductions in blood transfusions or $14 \%(n=2)[20,23]$ reported red cell units transfused per patient. The remaining three studies reported red cell units per 1000 patient days [16, 18], and number of patients transfused [17].

\section{Study quality}

Study quality was generally moderate (between 50 and 100\%) [40] (Table 1). We did not exclude studies based on quality as this was a descriptive review with no intent for meta-analysis, thus facilitating the investigation of quality issues in the literature. Generally, quality scores were lower due to a failure to provide transparent and detailed demographics, lack of discussion about the measurement instrument or where the designs were uncontrolled. There was also considerable variation in the length of follow up, and in some studies, there was a significant disparity in size between control and intervention groups.

\section{Barriers}

The barriers identified within each paper and the implementation strategies used to address them are summarised in Table 2. Eleven of the 39 CFIR constructs were identified as barriers to implementation including access to knowledge and information $(n=7)[10,12,13$, $16,18,21,22]$, knowledge and beliefs about the intervention $(n=7)[12,14-16,19,20,23]$, tension for change $(n=6)[10,14,15,17,18,21]$, culture $(n=4)$ $[12,15,20,23]$, structural characteristics $(n=4)[11,12$, $17,18]$, evidence strength and quality $(n=3)$ [12, 16, 20], available resources $(n=1)$ [18], complexity $(n=1)$ [18], engagement $(n=1)$ [18], peer pressure $(n=1)$ [20] and relative advantage $(n=1)$ [20]. Across the papers, a median of three barriers were reported, ranging between one and six.

\section{Implementation strategies}

Twenty-five different implementation strategies were identified in the included studies. The 10 most common implementation strategies were the following: conduct educational meetings (8 studies) [11, 12, 15, 17-19, 21, 23], audit and provide feedback (6 studies) [10-12, 15, $20,23]$, develop educational materials (6 studies) [10, 13, $15,17,20,21]$, conduct local consensus discussions (6 studies) [10,13,17, 18, 20, 23], develop and implement tools for quality monitoring (5 studies ) $[10,11,16,19$, $21]$, remind clinicians (5 studies) [11, 12, 14, 15, 22], involve executive boards (4 studies) [12, 18, 20, 22], distribute educational materials (3 studies) $[16,20,22]$, facilitate relay of clinical data to providers (3 studies) $[11,19,21]$, capture and share local knowledge (2 studies) $[15,18]$. Across the papers, a median of five implementation strategies were reported, ranging between one and seven.

\section{ERIC agreement}

Table 2 provides a summary of barriers reported and implementation strategies used in each paper and agreement with the ERIC recommendations. Six studies had over $80 \%$ agreement, five studies had 50\% agreement, one study had $20 \%$ agreement, and two studies had no agreement. The median and mode agreement was 50\%. Table 3 reports the individual barrier constructs, implementation strategies used to address them and the agreement with the ERIC recommendations. Implementation strategies in italic text indicate utilisation by the relevant study.

\section{Discussion}

This paper is the first to investigate and report barriers to implementation of the PBM guidelines and compare implementation strategies used with those recommended in the ERIC tool (measured as a level of agreement) [33]. Several key findings (in the context of PBM guidelines) became evident during the review. Firstly, only one paper reported the use of an implementation strategy, demonstrating poor knowledge, understanding 


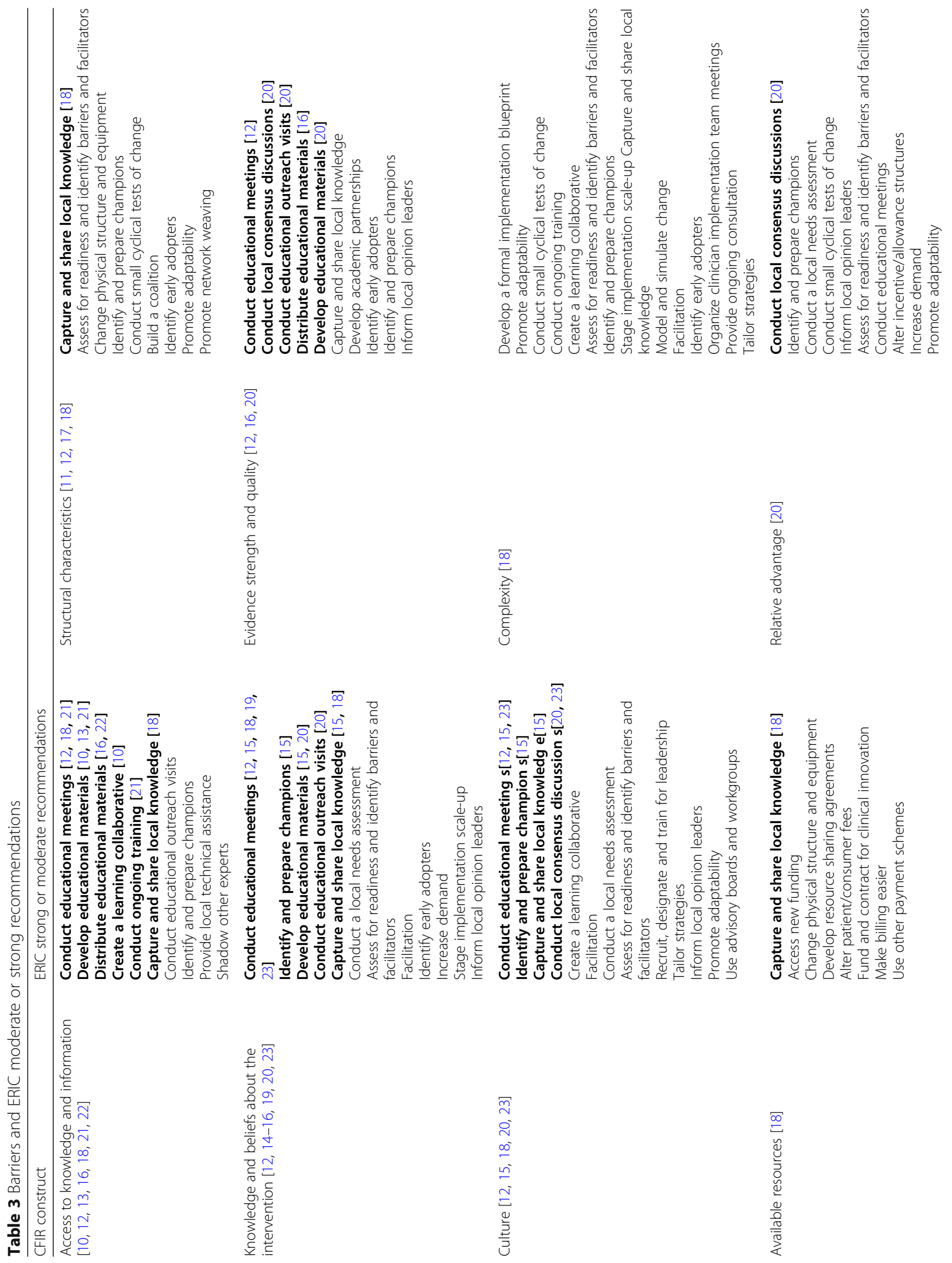




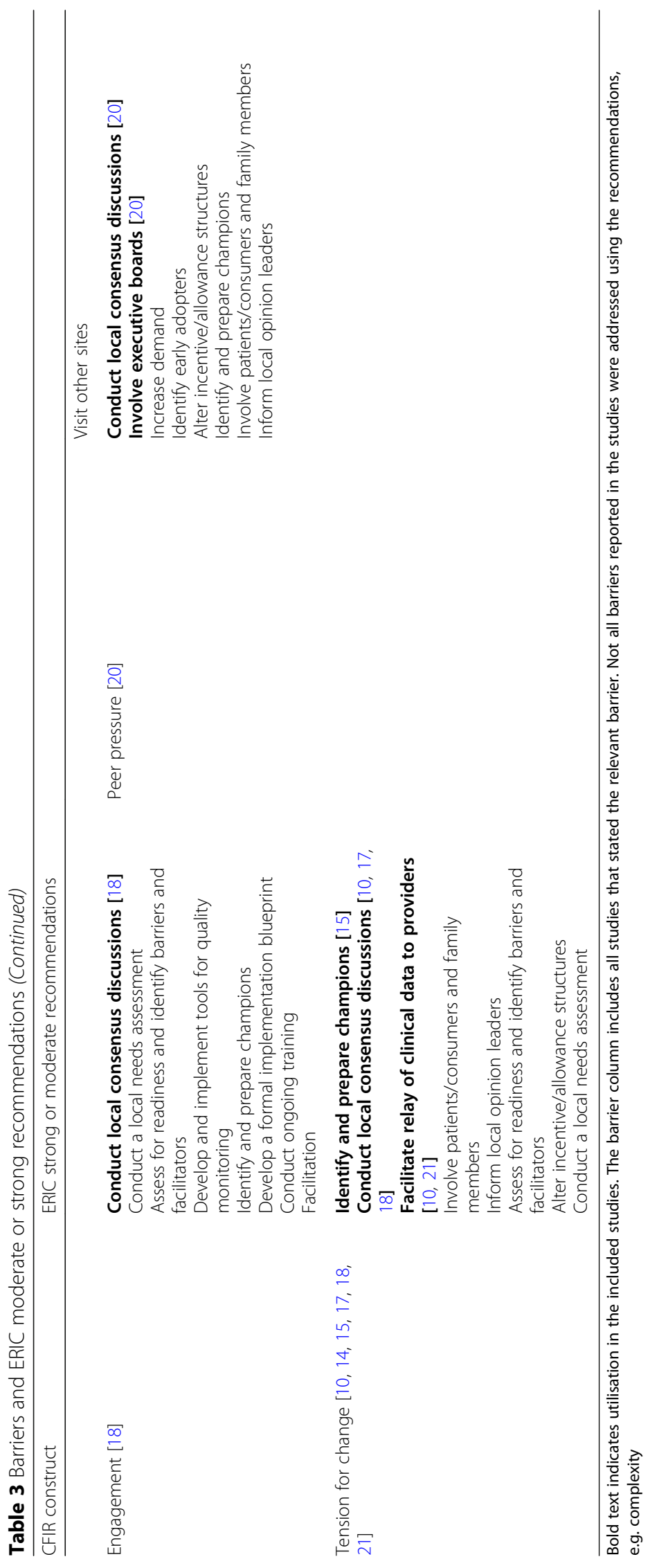


and application of implementation theory and frameworks in general. Secondly, the reporting of implementation studies is weak and requires improvement. While there was a cluster of barriers that were common amongst studies, there was high heterogeneity in the implementation strategies used. Finally, the ERIC tool can be used to provide guidance but requires further work to ascertain a strong consensus for recommended implementation strategies across all barriers.

Despite the existence of multiple theories and frameworks to help guide barrier identification, intervention selection and implementation process, only one study referred to a formal implementation theory, model or framework or existing quality improvement methods, although the authors did not explicitly state what it was [15]. This problem is not unique to PBM, and a recent review by Wensing and Grohl highlights the lack of theoretically informed implementation as a wider issue within implementation science [27]. Part of the problem may be the sheer number of tools available, and also, the knowledge required to identify and apply them appropriately [26]. As a result, the literature provides generalised reports about the effect of implementation strategies in local settings and fails to explain and report any implementation preparation undertaken or provide explicit detail as to the context in which the implementation occurred [12-14, 16-21, 23, 41]. The absence of a reported methodological approach presents a missed opportunity to test the effectiveness of implementation attempts rigorously. Future research should utilise available implementation methodologies to help improve the understanding of how to translate evidence to practice.

The quality of reporting of included studies was generally low and supports observations made by Luoto and colleagues that standards to help improve reporting quality in implementation studies are needed [42]. Standards that provide guidance are available, for example, the Standards for Reporting Implementation Studies (StaRI) [43], and they should be used routinely. The StaRI guidelines provide recommendations for both reporting an intervention and the associated implementation strategy [43]. The utilisation of the standards and improved reporting will provide the foundations for the validation and advancement of implementation theory, both in terms of describing interventions and the strategies used to implement them [42, 43]. All articles included focused on reporting the impact of implementation strategies used, and only one reported on an implementation model or theory but explained it only as having used "standard quality improvement methodologies" [15]. Just over half of the included studies attempted to tailor intervention strategies to their relevant context using local consensus processes $[10,13,17,18,20,23]$. It is advisable to ensure that implementation strategies are tailored to ensure compatibility with existing processes and acceptability of staff. A relevant example in the context of PBM is using the strategy of audit and feedback to help clinicians identify opportunities for improving practice. As recently outlined in a systematic review by Brown and colleagues, tailoring audits to local context is crucial to ensure the success of audit and feedback [44].

There was a cluster of common barriers reported in the included studies. However, in-depth explanation and exploration of barriers were limited in most articles, perhaps as the focus of the research was on describing the implementation strategies and how well they worked (i.e. reduction in red blood cell utilisation). The most common barriers reported in the studies were knowledge and beliefs about the intervention, access to knowledge and information, and tension for change. Knowledge and beliefs about the intervention was a barrier in seven papers and is defined as the individual's attitude and a general understanding of the key principles of an intervention [33]. Many papers acknowledge that this was a barrier for their facility and used various implementation strategies to educate their staff about their local PBM guidelines and why they are essential for patient safety. Interventions to support the implementation strategies included distributing information (e.g. pamphlets) [16, 22], setting up online learning portals [16], holding educational sessions at grand rounds [11], and implementation of performance tracking dashboards $[45,46]$. ERIC strategies that were not used to address this barrier included identifying local barriers, conducting a needs assessment and informing local opinion leaders. The utilisation of these implementation strategies to address the knowledge and belief barrier may enhance implementation efforts [33].

Access to knowledge and information was a barrier in seven papers and is defined as the availability of resources that provide education and guidance to support the uptake of an intervention [29]. Reporting of access to knowledge and information as a barrier included the acknowledgement of the absence of a contemporary local protocol or policy to guide transfusion decisionmaking [13]. The development of policy and procedure using local consensus discussions was undertaken in some instances [15]. The changes were then disseminated through educational meetings [12, 18, 21]. ERIC strategies that were not used to address this barrier centred around pragmatic educational implementation strategies, including conducting educational outreach visits, providing technical assistance and shadowing experts. The utilisation of these implementation strategies to address the access to knowledge and information barrier may enhance implementation efforts [33].

The tension for change (or rather, absence of) was a barrier in six papers, and this refers to the degree to which stakeholders perceive that change as necessary 
[29]. Reporting of tension for change included identified variability in practice, ignorance of best practice guidelines and current hospital performance $[10,14,15,17$, $18,21]$. The variability and lack of awareness was compounded by outdated practices, proliferated through myths held by some senior physicians, (for example, the dictum "if you are going to transfuse, you might as well use two units"), based on the premise that one unit was never adequate [12, 16]. Audit and feedback [10, 15] were utilised to provide clinicians with insight into their practice as well as the conduct of educational meetings $[15,17,18,21]$ to educate clinical staff on what is considered best practice. ERIC strategies that were unused included involving consumers and family, conducting local needs assessments, informing local opinion leaders, assessing barriers and altering incentive structures. The utilisation of these implementation strategies to address the tension for change barriers may enhance implementation efforts.

There was a high variation in the implementation strategies used in the included studies, with 25 different implementation strategies employed across the papers. Recent research undertaken by Althoff and colleagues included a meta-analysis of the effect of multimodal patient blood management programs and noted high heterogeneity of implementation strategies, supporting this finding [8]. Their review analysed implementation strategies used and their impact on red blood cell transfusion reduction but did not seek to understand the barriers faced by health professionals [8]. Health professionals would benefit from more explicit guidance as to which implementation strategies would best suit their local context. In order to use the data summarised in this paper, health professionals should use an implementation model or framework (e.g. CFIR) to help identify local barriers to see what has worked before, in the context of what is recommended by the ERIC tool [33]. Future research should focus on testing well-described implementation strategies, tailored to the local context.

The authors of the ERIC tool have commented that there was surprising heterogeneity between consensus for implementation strategies and acknowledge that further work is required to advance the utility of the tool [33]. The ERIC tool provided recommendations for ten of the barriers that were present in the included study [33]. One barrier had no moderate or strong recommendations, which was complexity, although this construct was only identified in one paper. Further refinement of the tool and the conduct of PBM implementation studies that utilise rigorous implementation science methodologies such as the ERIC tool, with quality reporting processes are needed to provide further guidance.
This review has several important limitations, the first of which is that many of the included papers were not written with the intent of reporting or analysing local barriers and implementation strategies that were used to address them. A large number of papers $(n=62)$ that would have been useful in terms of understanding the implementation strategies used and their impact on practice improvement were excluded because they did not explicitly mention existing barriers. The final limitation is the quality of reporting of interventions and implementation strategies used in the papers, which was generally quite poor. It is difficult to know if every implementation strategy and every intervention was mentioned in the papers, and this may have impacted on the ERIC agreement. We also acknowledge that the restricted review method chosen has potential limitations as we did not search for grey literature and the use of pilot sampling during screening means that we cannot be certain that all relevant literature was included.

\section{Conclusion}

The results of this review identified a cluster of barriers within PBM guideline implementation that consisted of 11 of 39 CFIR constructs. Despite the common barriers, there was high heterogeneity in the implementation strategies used by health professionals, with over 25 utilised. The most common barriers reported in the studies were knowledge and beliefs about the intervention, access to knowledge and information and tension for change. Common implementation strategies selected to address the barriers included conducting educational meetings, auditing and providing feedback, the development of educational materials, and conducting local consensus discussion. Health professionals should find these implementation strategies useful for addressing barriers to evidence-based patient blood management practice. Only one paper provided an explicit reference to having used an implementation model or framework, but it appears that in many (not all) instances, included papers were able to identify, and subsequently address most barriers, with the majority of studies demonstrating strong agreement with the ERIC tool. The utilisation of implementation frameworks and complementary tools may have enhanced this process. Studies need to utilise and report on implementation frameworks and tools to advance the field. Further refinement of the ERIC tool to include strong recommendations for all barriers would be advantageous in assisting health care professionals in selecting appropriate implementation strategies.

\section{Abbreviations}

CFIR: Consolidated Framework for Implementation Research; ERIC: Expert Recommendations for Implementing Change; PBM: Patient blood 
management; PRISMA: Preferred reporting items for systematic review and meta-analysis protocols); StaRI: Standards for Reporting Implementation studies

\section{Acknowledgements}

The author AD gratefully acknowledges assistance received from the Australian Government Research Training Program Scholarship.

\section{Authors' contributions}

AD contributed to the study design, all data collection, coding and synthesis, and the compilation of the manuscript. JD assisted with the conception and shaping of the study design, coding of data and reviewing the manuscript. JM assisted with the coding of the data and reviewing the manuscript. JM assisted with selecting the review method. JH assisted with reviewing the manuscript. All authors read and approved the final manuscript.

\section{Authors' information}

This review has been undertaken with the intent of inclusion in the author AD's Doctor of Philosophy Thesis.

\section{Funding}

Not applicable.

\section{Availability of data and materials}

All data generated or analysed during this study are included in this published article.

\section{Ethics approval and consent to participate}

Not applicable.

\section{Consent for publication}

Not applicable.

\section{Competing interests}

The authors declare that they have no competing interests

\section{Author details}

${ }^{1}$ The University of Newcastle, School of Nursing and Midwifery, University Drive, Callaghan, NSW 2302, Australia. ${ }^{2}$ School of Nursing/Institute for Health and Biomedical Innovation, Queensland University of Technology, Victoria Park Rd, Kelvin Grove, QLD 4059, Australia. ${ }^{3}$ Faculty of Health and Sports Sciences, The University of Agder, Grimstad, Norway. ${ }^{4}$ Mater Health Services, Level 6, Duncombe Building, Raymond Terrace, QLD 4101, Australia.

\section{Received: 14 August 2019 Accepted: 6 January 2020}

\section{Published online: 17 January 2020}

\section{References}

1. Isbister JP, Shander A, Spahn DR, et al. Adverse blood transfusion outcomes: establishing causation. Transfus Med Rev. 2011;25(2):89-101.

2. Carson JL, Triulzi DJ, Ness PM. Indications for and adverse effects of red-cell transfusion. N Engl J Med. 2017;377(13):1261-72.

3. Poles D, et al. On behalf of the Serious Hazards of Transfusion (SHOT) Steering Group. In: Bolton-Maggs, editor. The 2017 Annual SHOT Report. 2018.

4. Munoz M, Acheson AG, Auerbach $M$, et al. International consensus statement on the peri-operative management of anaemia and iron deficiency. Anaesthesia. 2017;72(2):233-47.

5. Vaglio S, Gentili S, Marano G, et al. The italian regulatory guidelines for the implementation of patient blood management. Blood Transfus. 2017;15(4): 325-8.

6. National Blood Authority and National Health Medical Research Council, Patient blood management guidelines: Module 2 - perioperative. Canberra: National Blood Authority; 2012.

7. Gammon HM, Waters JH, Watt A, et al. Developing performance measures for patient blood management. Transfusion. 2011;51(11):2500-9.

8. Althoff FC, Neb H, Herrmann E, et al. Multimodal patient blood management program based on a three-pillar strategy: A systematic review and meta-analysis. Ann Surg. 2019;269(5):794-804.

9. Meybohm P, Fischer DP, Weber CF, et al. Safety and effectiveness of a patient blood management programme in surgical patients - the study design for a multicentre epidemiological non-inferiority trial by the german pbm network. ISBT Science Series. 2015;10(S1):141-5.

10. Whitney G, Daves S, Hughes A, et al. Implementation of a transfusion algorithm to reduce blood product utilization in pediatric cardiac surgery. Paediatr Anaesth. 2013;23(7):639-46.

11. Zuckerberg GS, Scott AV, Wasey JO, et al. Efficacy of education followed by computerized provider order entry with clinician decision support to reduce red blood cell utilization. Transfusion. 2015;55(7):1628-36.

12. Abbett SK, Kaufman RM, Gustafson M, et al. Multifaceted approach to reducing unnecessary red blood cell utilization. Healthcare. 2015;3(2):67-73.

13. Albinarrate A, Lopez-Picado A, Oiartzabal I, et al. Assessment of the introduction of a blood management program in orthopaedic surgery. Revista Espanola de Anestesiologia y Reanimacion. 2015;62(8):443-9.

14. Ansari S, Szallasi A. Blood management by transfusion triggers: When less is more. Blood Transfus. 2012:10(1):28-33.

15. Brevig J, McDonald J, Zelinka ES, et al. Blood transfusion reduction in cardiac surgery: Multidisciplinary approach at a community hospital. The Annals of Thoracic Surgery. 2009;87(2):532-9.

16. Cohn CS, Welbig J, Bowman R, et al. A data-driven approach to patient blood management. Transfusion. 2014;54(2):316-22.

17. Garrioch M, Sandbach J, Pirie E, et al. Reducing red cell transfusion by audit, education and a new guideline in a large teaching hospital. Transfus Med. 2004;14(1):25-31.

18. Kumar A, Figueroa PI, Gowans $\mathrm{KL}$, et al. An evolution in blood management Past, present, and future. Qual Manag Health Care. 2011;20(4):311-21.

19. Mallett SV, Peachey TD, Sanehi O, et al. Reducing red blood cell transfusion in elective surgical patients: The role of audit and practice guidelines. Anaesthesia. 2000;55(10):1013-9.

20. Oliver JC, Griffin RL, Hannon T, et al. The success of our patient blood management program depended on an institution-wide change in transfusion practices. Transfusion. 2014;54(10 Pt 2):2617-24.

21. Pearse BL, Smith I, Faulke D, et al. Protocol guided bleeding management improves cardiac surgery patient outcomes. Vox Sanguinis. 2015;109(3):267-79.

22. Rineau E, Chaudet A, Chassier C, et al. Implementing a blood management protocol during the entire perioperative period allows a reduction in transfusion rate in major orthopedic surgery: A before-after study. Transfusion. 2016:56(3):673-81.

23. Szpila BE, Ozrazgat-Baslanti T, Zhang J, et al. Successful implementation of a packed red blood cell and fresh frozen plasma transfusion protocol in the surgical intensive care unit. PLOS ONE [Electronic Resource]. 2015;10(5):e0126895.

24. Delaforce A, Moore D, Duff J, et al. Assessing transfusion practice in elective surgical patients: a baseline audit. ISBT Science Series. 2019; 14(4):415-22.

25. Tinmouth A, Macdougall L, Fergusson D, et al. Reducing the amount of blood transfused: A systematic review of behavioral interventions to change physicians' transfusion practices. Arch Intern Med. 2005;165(8):845-52.

26. Nilsen P. Making sense of implementation theories, models and frameworks. Implement Sci. 2015;10(1):53

27. Wensing M, Grol R. Knowledge translation in health: How implementation science could contribute more. BMC Medicine. 2019;17(1):88.

28. French SD, Green SE, O'Connor DA, et al. Developing theory-informed behaviour change interventions to implemen evidence into practice: $A$ systematic approach using the theoretical domains framework. Implement Sci. 2012;7(1):38

29. Damschroder LJ, Aron DC, Keith RE, et al. Fostering implementation of health services research findings into practice: A consolidated framework for advancing implementation science. Implement Sci. 2009;4(1):50

30. Graham I, Logan J, Harrison M, et al. Lost in knowledge translation: Time for a map? J Contin Educ Health Prof. 2006:26(1):13-24.

31. Breimaier HE, Heckemann B, Halfens RJG, et al. The consolidated framework for implementation research (cfir): A useful theoretical framework for guiding and evaluating a guideline implementation process in a hospital-based nursing practice. North America: BioMed Central: United States; 2015.

32. Keith RE, Crosson JC, O'Malley AS, et al. Using the consolidated framework for implementation research (cfir) to produce actionable findings: A rapidcycle evaluation approach to improving implementation. Implementation Science. 2017;12(1):1-12. 
33. Waltz TJ, Powell BJ, Fernández ME, et al. Choosing implementation strategies to address contextual barriers: Diversity in recommendations and future directions. Implementation Science. 2019;14(1):42.

34. Powell BJ, Waltz TJ, Chinman MJ, et al. A refined compilation of implementation strategies: Results from the expert recommendations for implementing change (eric) project. Implementation Science. 2015;10(1):21.

35. Pluddemann A, Aronson JK, Onakpoya I, et al. Redefining rapid reviews: A flexible framework for restricted systematic reviews. BMJ Evid Based Med. 2018;23(6):201-3.

36. Hebert PC, Wells G, Blajchman MA, et al. A multicenter, randomized, controlled clinical trial of transfusion requirements in critical care. Transfusion requirements in critical care investigators, canadian critical care trials group. N Engl J Med. 1999;340(6):409-17.

37. Victoor A, Delnoij DM, Friele RD, et al. Determinants of patient choice of healthcare providers: A scoping review. BMC Health Serv Res. 2012;12(1):272.

38. Pham MT, Rajić A, Greig JD, et al. A scoping review of scoping reviews: Advancing the approach and enhancing the consistency. Research Synthesis Methods. 2014;5(4):371-85.

39. Moher D, Liberati A, Tetzlaff J, et al. Preferred reporting items for systematic reviews and meta-analyses: The prisma statement. BMJ. 2009; 339:b2535.

40. Pluye, P, Robert, E., Cargo, M., Bartlett, G., O'Cathain, A., Griffiths, F., Boardman, F., Gagnon, MP., \& Rousseau, MC. Proposal: a mixed methods appraisal tool for systematic mixed studies reviews. 2011; Available from: http://mixedmethodsappraisaltoolpublic.pbworks.com . Archived by WebCite at http://www.webcitation.org/5tTRTc9yJ.

41. Rineau E, Stoyanov A, Samson E, et al. Patient blood management in major orthopedic surgery: Less erythropoietin and more iron? Anesth Analg. 2017; 125(5):1597-9.

42. Luoto J, Shekelle PG, Maglione MA, et al. Reporting of context and implementation in studies of global health interventions: A pilot study. Implementation Science. 2014;9(1):57.

43. Pinnock H, Barwick M, Carpenter CR, et al. Standards for reporting implementation studies (stari) statement. BMJ. 2017;356:16795.

44. Brown B, Gude WT, Blakeman T, et al. Clinical performance feedback intervention theory (cp-fit): A new theory for designing, implementing, and evaluating feedback in health care based on a systematic review and metasynthesis of qualitative research. Implementation Science. 2019;14(1):40.

45. Gomez AT, Quinn JG, Doiron DJ, et al. Implementation of a novel real-time platelet inventory management system at a multi-site transfusion service. Transfusion. 2015;55(9):2070-5.

46. Gallagher T, Leahy MF, Darby S, et al. Assembling a state-wide patient blood management program as a standard of care: The western australian experience. ISBT Science Series. 2017;12(3):365-74.

\section{Publisher's Note}

Springer Nature remains neutral with regard to jurisdictional claims in published maps and institutional affiliations.

\section{Ready to submit your research? Choose BMC and benefit from:}

- fast, convenient online submission

- thorough peer review by experienced researchers in your field

- rapid publication on acceptance

- support for research data, including large and complex data types

- gold Open Access which fosters wider collaboration and increased citations

- maximum visibility for your research: over $100 \mathrm{M}$ website views per year

At BMC, research is always in progress.

Learn more biomedcentral.com/submissions 